\title{
On the continuity of the polyconvex, quasiconvex and rank-one- convex envelopes with respect to growth condition
}

\author{
Wilfrid Gangbo \\ Ecole Polytechnique Fédérale de Lausanne, 1015 Lausanne, Switzerland
}

(MS received 29 April 1991. Revised MS received 24 June 1992)

\section{Synopsis}

Let $C f, P f, Q f$ and $R f$ be respectively the convex, polyconvex, quasi-convex and rank-one-convex envelopes of a given function $f$. If $f_{p}: \mathbb{R}^{N \times M} \mapsto \mathbb{R}$ and $f_{q}(\xi)$ behaves as $|\xi|^{q}$ at infinity $q \in(1, \infty)$, we show that $\lim _{p \rightarrow q} C f_{p}=C f_{q}, \lim _{p \rightarrow q} Q f_{p}=Q f_{q}, \lim _{p \rightarrow q} R f_{p}=R f_{q}$. This is the case for $\left(P f_{p}\right)_{p}$ provided that $q \neq 1, \ldots, \min (N, M)$, otherwise $\liminf _{p \rightarrow q} P f_{p} \neq P f_{q}$. In the last part of this work, we show that $f(\xi)=g(|\xi|)$ does not imply in general $P f=Q f$.

\section{Introduction}

Let $f_{p}: \mathbb{R}^{N \times M} \mapsto \mathbb{R}$ be a Borel measurable function which behaves at infinity as $|\xi|^{p}, p \geqq 1$. Let $C f_{p}, P f_{p}, Q f_{p}$ and $R f_{p}$ be, respectively, the convex, polyconvex, quasiconvex and rank-one-convex envelopes of $f_{p}$. (For a precise definition, see the end of the introduction.) We want to study the continuity with respect to $p$ of these envelopes. As is well known, they are discontinuous at $p=1$. We show that $C f_{p}, Q f_{p}$ and $R f_{p}$ are, however, continuous at $p>1$. (The result for $C f_{p}$ is elementary.) In the case of $P f_{p}$, we prove that it is discontinuous provided that $p=2, \ldots, \min (N, M)$, and otherwise continuous. We next give two examples, the first one being elementary.

Example 1.1. For $0<p<1$, let

$$
f_{p}(\xi)=|\xi|^{p}, \quad 0 \leqq p \leqq 1, \quad \xi \in \mathbb{R}^{N \times N} .
$$

We find that

$$
\begin{aligned}
\liminf _{p \rightarrow 1} C f_{p}(\xi) & =\liminf _{p \rightarrow 1} P f_{p}(\xi)=\liminf _{p \rightarrow 1} Q f_{p}(\xi)=\liminf _{p \rightarrow 1} R f_{p}(\xi) \equiv 0 \\
& <C f_{1}(\xi)=P f_{1}(\xi)=Q f_{1}(\xi)=R f_{1}(\xi)=|\xi| .
\end{aligned}
$$

EXAMPLE 1.2. Recall first that a polyconvex function with a subquadratic growth is necessarily convex. (See Remark 3.5 .) For $1 \leqq p<2, \xi \in \mathbb{R}^{2 \times 2}$, let

$$
f_{p}(\xi)=\left\{\begin{array}{lll}
1+|\xi|^{p} & \text { if } & |\xi| \neq 0 \\
0 & \text { if } & |\xi|=0
\end{array}\right.
$$

In view of the above remark, we find $P f_{p}=C f_{p}$ for every $1 \leqq p<2$. Kohn and Strang in [9] proved that $R f_{2}=Q f_{2}=P f_{2}$ and $C f_{2}(\xi) \neq P f_{2}(\xi)$ if and only if 
$0<|\xi|^{2}+2|\operatorname{det}(\xi)|<1$ and $\operatorname{det}(\xi) \neq 0$. Computing $C f_{p}$, and using the result of Kohn and Strang:

$$
\liminf _{p \rightarrow 2} P f_{p} \neq P f_{2} .
$$

We now describe the contents of this paper. In Section 2 we show an elementary result: for every $q \in(1, \infty), \lim _{p \rightarrow q} C f_{p}=C f_{q}$. In Section 3 we show that:

(i) for every $q \in(1, \infty), q \neq 2, \ldots, \min (N, M) \lim _{p \rightarrow q} P f_{p}=P f_{q}$;

(ii) in some examples the result is false provided that $q \in\{2, \ldots, \min (N, M)\}$.

In Section 4 we prove that for every $q \in(1, \infty), \lim _{p \rightarrow q} Q f_{p}=Q f_{q}$. To achieve this, we first approximate $Q f_{p}(\xi)$ by $1 /|Q| \int_{Q} f_{p}\left(\xi+\nabla \phi^{p}\right)$, where $\phi^{p} \in W_{0}^{1, p}(Q)^{M}$. (See [4].) The proof is based on Gehring's lemma on reverse Hölder inequality in [6] and a result of Giaquinta and Modica in [7]. We deduce, as a byproduct, that there exist quasiconvex functions $f: \mathbb{R}^{N \times M} \mapsto \mathbb{R}$ for every $N, M>1$ integers, that are not polyconvex. (See $[\mathbf{1 4}, \mathbf{1}]$.) We also obtain a general method of constructing such functions. We conclude this section by studying some examples such as:

$$
f_{p}(\xi)=|\xi|^{p}+a|\operatorname{det}(\xi)|^{p / 2}, \quad a>0,
$$

and we prove that $P f_{p} \neq Q f_{p}$ for $p$ near 2 .

In Section 5, we show that for every $q \in(1, \infty), \lim _{p \rightarrow q} R f_{p}=R f_{q}$. To achieve this, we make an additional hypothesis on the family of functions $\left(f_{p}\right)_{p}$ and assume that there exists a constant $K>0$ such that $R f_{p}(\xi)=f_{p}(\xi)$ for every $|\xi| \geqq K$. In some examples this is satisfied. In Section 6 , we turn our attention to the following question: if $f$ is a function such that $f(\xi)=g(|\xi|)$, does this always imply that Pf $=Q f$ ? Note that in the example of Kohn and Strang (Example 1.2 above) $P f_{2}=Q f_{2}$. We show that, in general, if $p<2$ then $P f_{p}<Q f_{p}$. Here, we use an interesting method (similar to one of Boccardo and Gallouet in an article in preparation) to obtain strong convergence of a certain weakly convergent sequence.

We conclude this introduction by giving some definitions used above.

Definitions 1.3 (see [4]). Let $f: \mathbb{R}^{N \times M} \mapsto \mathbb{R}$ be a Borel measurable function

(a) $f$ is said to be convex if $f(\lambda \xi+(1-\lambda) \eta) \leqq \lambda f(\xi)+(1-\lambda) f(\eta)$ for every $\xi, \eta \in \mathbb{R}^{N \times M}$ and every $\lambda \in(0,1)$.

(b) $f$ is said to be polyconvex if there exists a function $h: \mathbb{R}^{\tau(N, M)} \mapsto \mathbb{R}$, convex such that $f(\xi)=h(T(\xi))$ for every $\xi \in \mathbb{R}^{N \times M}$, where $\tau(N, M)=\sum_{1 \leqq s \leqq \min (N, M)}$ $\left(\begin{array}{c}M \\ s\end{array}\right)\left(\begin{array}{c}N \\ s\end{array}\right), T(\xi)=\left(a d j_{1} \xi, \ldots, a d j_{\min (\mathrm{N}, \mathrm{M})} \xi\right)$ and $a d j_{s} \xi$ stands for the matrix of all $s \times s$ minors of $\xi$. If $N=M=2$ then $T(\xi)=(\xi, \operatorname{det}(\xi))$.

(c) $f$ is said to be quasiconvex if $\frac{1}{|\Omega|} \int_{\Omega} f(\xi+\nabla \phi) \geqq f(\xi)$ for every $\xi \in \mathbb{R}^{N \times M}$, every $\Omega \subset \mathbb{R}^{N}$ (or equivalently for some $\Omega \subset \mathbb{R}^{N}$ ) and every $\phi \in W_{0}^{1, \infty}(\Omega)^{M}$. 
(d) $f$ is said to be rank-one-convex if $f(\lambda \xi+(1-\lambda) \eta) \leqq \lambda f(\xi)+(1-\lambda) f(\eta)$ for every $\xi, \eta \in \mathbb{R}^{N \times M}$ with $\operatorname{rank}(\xi-\eta) \leqq 1$ and every $\lambda \in(0,1)$.

It is a well-established fact, following the work of Morrey $[11,12]$ and later of Ball [2] that, in general, one has:

$$
f \text { convex } \Rightarrow f \text { polyconvex } \Rightarrow f \text { quasiconvex } \Rightarrow f \text { rank-one-convex. }
$$

The different envelopes are defined as:

$$
\begin{aligned}
& C f=\sup \{g, g \leqq f, g \text { convex }\}, \\
& P f=\sup \{g, g \leqq f, g \text { polyconvex }\}, \\
& Q f=\sup \{g, g \leqq f, g \text { quasiconvex }\}, \\
& R f=\sup \{g, g \leqq f, g \text { rank-one-convex }\} .
\end{aligned}
$$

\section{Continuity of $C f_{p}$ with respect to $p$}

We start with the main result of this section.

TheOREM 2.1. Let $[\alpha, \beta] \subset(1, \infty), F, G, \gamma_{0}>0, C \geqq 1 N, M \geqq 1$ be two integers. Let $w:[0, \infty) \mapsto[0, \infty)$ with $\lim _{t \rightarrow 0} w(t)=w(0)=0, \quad \sup \{w(t), t \in[0, \beta]\} \leqq G$ and $f_{p}: \mathbb{R}^{N \times M} \mapsto \mathbb{R}$ lower semicontinuous, $p \in[\alpha, \beta]$, such that:

$$
\begin{gathered}
|\xi|^{p} \leqq f_{p}(\xi) \leqq C\left(1+|\xi|^{p}\right) \quad \text { for every } p \in[\alpha, \beta] \text { and every } \xi \in \mathbb{R}^{N \times M}, \\
\left|f_{p}(\xi)-f_{q}(\xi)\right| \leqq \frac{F}{\gamma} w(p-q)\left(1+|\xi|^{p+\gamma}\right) \text { for every } \gamma \in\left(0, \gamma_{0}\right), \\
\text { every } \xi \in \mathbb{R}^{N \times M}, \text { and every } p, q \in[\alpha, \beta] \text { with } p>q
\end{gathered}
$$

Then

$$
\lim _{p \rightarrow q} C f_{p}(\xi)=C f_{q}(\xi), \quad \text { for every } \xi \in \mathbb{R}^{N \times M} \text { and every } q \in(\alpha, \beta) .
$$

Before we prove this theorem, let us begin with some remarks.

Remarks 2.2. (a) In general, we have $\lim _{p \rightarrow 1^{-}} C f_{p}<C f_{1}$. Indeed, if $f_{p}(\xi)=$ $|\xi|^{p}, \xi \in \mathbb{R}^{N \times M}$, then $0 \equiv \lim _{p \rightarrow 1^{-}} C f_{p}<C f_{1}=f_{1}$.

(b) Theorem 2.1 is still true if we replace the condition (2.1) by $a+b|\xi|^{p} \leqq f_{p}(\xi) \leqq C\left(1+|\xi|^{p}\right)$ where $a \in \mathbb{R} b>0$ are two constants.

(c) To prove (2.3), we will show that $\left|C f_{p}(\xi)-C f_{q}(\xi)\right|$ and $\left|f_{p}(\xi)-f_{q}(\xi)\right|$ have the same modulus of continuity.

ExAMPLEs 2.3. The following examples satisfy the hypotheses of the theorem:

$$
\begin{gathered}
f_{p}(\xi)=\left\{\begin{array}{lll}
1+|\xi|^{p} & \text { for } & |\xi| \neq 0, \\
0 & \text { for } & |\xi|=0,
\end{array} \quad \xi \in \mathbb{R}^{N \times M} ;\right. \\
f_{p}(\xi)=|\xi|^{p}+a|\operatorname{det}(\xi)|^{p / N}, \quad \xi \in \mathbb{R}^{N \times N}, \quad a>0 .
\end{gathered}
$$


We get that $\left(f_{p}\right)_{p}$ verifies (2.1) and (2.2). Hence Theorem 2.1 leads to $\lim _{p \rightarrow q} C f_{p}=C f_{q}$, for every $q>1$.

To prove Theorem 2.1, we begin with an elementary lemma.

Lemma 2.4. Let $N, M \geqq 1$ be two integers, $\alpha, \beta \in(1, \infty), C>0$ a constant and $f: \mathbb{R}^{N \times M} \mapsto \mathbb{R}$ lower semicontinuous such that:

$$
|\xi|^{p} \leqq f_{p}(\xi) \leqq C\left(1+|\xi|^{p}\right) \text { for some } p \in[\alpha, \beta] \text { and every } \xi \in \mathbb{R}^{N \times M} .
$$

Then, there exists a constant $D>0$ depending only on $\alpha, \beta$ and $C$ such that, for every $\xi, \xi^{*}, \eta \in \mathbb{R}^{N \times M}$,

$$
C f(\eta)=\left\langle\eta, \xi^{*}\right\rangle-f^{*}\left(\xi^{*}\right), \quad C f(\xi)=\left\langle\xi, \xi^{*}\right\rangle-f^{*}\left(\xi^{*}\right) \quad \text { implies } \quad|\eta| \leqq D(1+|\xi|) .
$$

Proof. Using (2.1), we get that, for every $\xi^{*} \in \mathbb{R}^{N \times M}$,

$$
C \sup \left\{0, \frac{p-1}{(C p)^{\hat{p}}}\left|\xi^{*}\right|^{\hat{p}}-1\right\} \leqq f^{*}\left(\xi^{*}\right) \leqq \frac{p-1}{(p)^{\dot{p}}}|\xi|^{\dot{p}}, \quad \text { where } \quad \tilde{p}=\frac{p}{p-1} .
$$

Adding (2.1) and (2.4), we deduce that there exists a constant $E>0$ such that

$$
\left|\xi^{*}\right| \leqq E(1+|\xi|) \text {. }
$$

Then (2.1) implies that there exists an $s \in[0,1]$ such that

$$
C f(\eta)=\left\langle\eta, \xi^{*}\right\rangle-f^{*}\left(\xi^{*}\right)=s|\eta|^{p}+(1-s)\left(1+|\eta|^{p}\right) .
$$

Hence

$$
\left.|| \eta\right|^{p-1}\left(s+(1-s) C-\left\langle\frac{\eta}{|\eta|} ; \xi^{*}\right\rangle \mid\right)|\leqq|(1-s) C+\left.f^{*}\left(\xi^{*}\right)\right|^{(p-1) / p},
$$

or

$$
|\eta| \leqq\left|(1-s) C+f^{*}\left(\xi^{*}\right)\right|^{1 / p} .
$$

Adding (2.5) and (2.6) to these previous inequalities, we conclude the proof.

We now prove Theorem 2.1.

Proof of Theorem 2.1. Let $\xi \in \mathbb{R}^{N \times M} ;(2.1)$ implies that there exists

such that

$$
\lambda_{1}^{p}, \ldots, \lambda_{N M+1}^{p} \in[0,1], \quad \xi_{1}^{p}, \ldots, \xi_{N M+1}^{p} \in \mathbb{R}^{N \times M}
$$

$$
\sum_{i=1}^{N M+1} \lambda_{i}^{p}=1, \quad \sum_{i=1}^{N M+1} \lambda_{i}^{p} \xi_{i}^{p}=\xi \quad \text { and } \quad \sum_{i=1}^{N M+1} \lambda_{i}^{p} f_{p}\left(\xi_{i}^{p}\right)=C f_{p}(\xi)
$$

Let $\xi^{*} \in \mathbb{R}^{N \times M}$ such that $C f_{p}(\xi)=\left\langle\xi, \xi^{*, p}\right\rangle-f_{p}^{*}\left(\xi^{*, p}\right)$. It is obvious that

$$
C f_{p}\left(\xi_{i}^{p}\right)=f_{p}\left(\xi_{i}^{p}\right) \text { and } C f_{p}\left(\xi_{i}^{p}\right)=\left\langle\xi_{i}^{p}, \xi^{*, p}\right\rangle-f_{p}^{*}\left(\xi^{*, p}\right), \quad i=1, \ldots, N M+1 .
$$


By Lemma 2.4, we find that there exists a constant $D>0$ depending only on $\alpha, \beta, C$ such that

$$
\left|\xi_{i}^{p}\right| \leqq D(1+|\xi|) i=1, \ldots, N M+1 \quad \text { for every } p \in[\alpha, \beta] .
$$

Then we conclude that there exists a constant $H>0$ depending only on $\alpha, \beta, C$ such that

$$
\left|C f_{p}(\xi)-C f_{q}(\xi)\right| \leqq \frac{H}{\gamma} w(p-q)\left(1+|\varepsilon|^{p+\gamma}\right) \quad \text { for every } \quad \gamma \in\left(0, \gamma_{0}\right) .
$$

Hence Theorem 2.1 is proved.

\section{Continuity and discontinuity of $\boldsymbol{P} f_{p}$ with respect to $\boldsymbol{p}$}

We start with the main result of this section.

Theorem 3.1. Let $[\alpha, \beta] \subset(1, \infty), F, G, \gamma_{0}>0, C \geqq 1$ and $N, M>1$ be two integers. Let $w:[0, \infty) \mapsto[0, \infty)$ with $\lim _{t \rightarrow 0} w(t)=w(0)=0, \sup \{w(t), t \in[0, \beta]\} \leqq G$ and $f_{p}: \mathbb{R}^{N \times M} \mapsto \mathbb{R}$ lower semicontinuous, $p \in[\alpha, \beta]$, such that:

$$
\begin{gathered}
|\xi|^{p} \leqq f_{p}(\xi) \leqq C\left(1+|\xi|^{p}\right) \quad \text { for every } \quad p \in[\alpha, \beta] \text { and every } \xi \in \mathbb{R}^{N \times M} ; \\
\left|f_{p}(\xi)-f_{q}(\xi)\right| \leqq \frac{F}{\gamma} w(p-q)\left(1+\mid \xi^{p+\gamma}\right) \text { for every } \gamma \in\left(0, \gamma_{0}\right)
\end{gathered}
$$

every $\xi \in \mathbb{R}^{N \times M}$, and every $p, q \in[\alpha, \beta]$ with $p>q$. Then

$$
\text { in general } \lim _{p \rightarrow q} P f_{p}<P f_{q}, \text { for } q=2, \ldots, \min (N, M) \text {; }
$$

$\lim _{p \rightarrow q} P f_{p}(\xi)=P f_{q}(\xi), \quad$ for every

$$
\xi \in \mathbb{R}^{N \times M} \text { and every } q \in(\alpha, \beta) q \neq 2, \ldots, \min (N, M) .
$$

Before proving Theorem 3.1, let us begin with some remarks.

Remarks 3.2. (a) In general, we also have $\lim _{p \rightarrow 1^{-}} P f_{p}<P f_{1}$. Indeed, if $f_{p}(\xi)=|\xi|^{p}, \xi \in \mathbb{R}^{N \times M}$, then $0 \equiv \lim _{p \rightarrow 1^{-}} P f_{p}<P f_{1}=f_{1}$.

(b) Theorem 3.1 is still true if we change the condition (3.1) to $a+b|\xi|^{p} \leqq f_{p}(\xi) \leqq C\left(1+|\xi|^{p}\right)$, where $a \in \mathbb{R}, b>0$ are two constants.

Notation. For $\xi \in \mathbb{R}^{N \times M}, q \in \mathbb{N}, \operatorname{adj}_{q}(\xi)$ stands for the matrix of all $q \times q$ minors of $\xi$. (3.5) 
ExAmPLes 3.3. (1) Let $f_{p}(\xi)=|\xi|^{p}+a\left|a d j_{q}(\xi)\right|^{p / q}, \quad \xi \in \mathbb{R}^{N \times N}, \quad a>0$ and $q \in\{2,3,4, \ldots\}$. We get that $\left(f_{p}\right)_{p}$ verifies (3.1) and (3.2). Hence Theorem 3.1 leads to $\lim _{p \rightarrow q} P f_{p}=P f_{q}$, if $q \neq 2, \ldots, \min (N, M)$. We show (see Step 4 of the proof of Theorem 3.1) that $\lim _{p \rightarrow q} P f_{p}<P f_{q}$ for suitable values of $a$.

(2) Let

$$
f_{p}(\xi)=\left\{\begin{array}{lll}
1+|\xi|^{p} & \text { for } & |\xi| \neq 0, \\
0 & \text { for } & |\xi|=0,
\end{array} \quad \xi \in \mathbb{R}^{2 \times 2} .\right.
$$

We get that $\left(f_{p}\right)_{p}$ verifies (3.1) and (3.2). Hence Theorem 3.1 leads to $\lim _{p \rightarrow q} P f_{p}=P f_{q}$, for every $q>1, q \neq 2$.

Knowing that $P f_{p}=C f_{p}$ for every $0<p<2$ and $C f_{2}<P f_{2}$ (see [9]), we can deduce that $\lim _{p \rightarrow 2^{-}} P f_{p}<P f_{2}$. We also get that $\lim _{p \rightarrow 1^{-}} P f_{p} \equiv 0<P f_{1}=f_{1}$.

To prove Theorem 3.1, let us now begin with the following lemma:

Lemma 3.4. Let $N, M \geqq 1$ be two integers, $p \in[1, \min (N, M)], C>0$ a constant and $f: \mathbb{R}^{N \times M} \mapsto \mathbb{R}$ lower semicontinuous such that:

$$
|\xi|^{p} \leqq f_{p}(\xi) \leqq C\left(1+|\xi|^{p}\right) \quad \text { for every } \quad \xi \in \mathbb{R}^{N \times M} .
$$

Then the three following assertions are equivalent:

- $f$ is polyconvex;

- $\lambda_{1}, \ldots, \lambda_{r} \in[0,1], \xi_{1}, \ldots, \xi_{r} \in \mathbb{R}^{N \times M} \sum_{i=1}^{r} \lambda_{i}=1, \sum_{i=1}^{r} \lambda_{i} R\left(\xi_{i}\right)=R\left(\sum_{i=1}^{r} \lambda_{i} \xi_{i}\right)$

implies

$\sum_{i=1}^{r} \lambda_{i} f\left(\xi_{i}\right) \geqq f\left(\sum_{i=1}^{r} \lambda_{i} \xi_{i}\right)$,

where

$$
\begin{aligned}
R(\xi) & =\left(\operatorname{adj}_{1}(\xi), \ldots, \operatorname{adj}_{[p]}(\xi)\right), \quad \xi \in \mathbb{R}^{N \times M}, \\
r & =1+\sum_{i=1}^{[p]}\left(\begin{array}{c}
N \\
i
\end{array}\right)\left(\begin{array}{c}
M \\
i
\end{array}\right), \text { and }[p] \text { is the integer part of } p ;
\end{aligned}
$$

- $f(\xi)=h(R(\xi))$

where for

$$
\begin{array}{r}
X \in \mathbb{R}^{r-1}, h(X)=\inf \left\{\sum_{i=1}^{r} \lambda_{i} f\left(\xi_{i}\right), \lambda_{i} \in[0,1] \xi_{i} \in \mathbb{R}^{N \times M} i=1, \ldots, r \sum_{i=1}^{r} \lambda_{i}=1,\right. \\
\left.\sum_{i=1}^{r} \lambda_{i} R\left(\xi_{i}\right)=X\right\} .
\end{array}
$$


Proof. The proof of Lemma 3.4 is a direct adaptation of the proof of the representation theorem of the polyconvex envelope. (See $[4$, p. 201, Theorem 1.1]).

Remarks 3.4. (a) We can see that $h: \mathbb{R}^{r-1} \mapsto \mathbb{R}$ is convex.

(b) An immediate consequence of Lemma 3.4 is that a polyconvex function $f: \mathbb{R}^{N \times M} \mapsto \mathbb{R}$ with subquadratic growth is convex.

We now start with the proof of Theorem 3.1.

Proof of Theorem 3.1. We divide the proof into four steps. Let $\xi \in \mathbb{R}^{N \times M}$, and $p \in[\boldsymbol{\alpha}, \boldsymbol{\beta}]$.

Step 1. We prove here that $\lim \sup P f_{p}(\xi) \leqq P f_{q}(\xi)$. Let $\varepsilon>0$. There exist

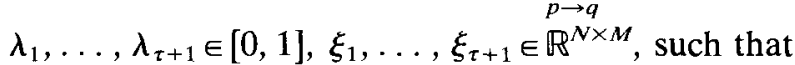

$$
\sum_{i=1}^{\tau+1} \lambda_{i}=1, \quad \sum_{i=1}^{\tau+1} \lambda_{i} T\left(\xi_{i}\right)=T(\xi) \text { and } \sum_{i=1}^{\tau+1} \lambda_{i} f_{q}\left(\xi_{i}\right)<-\varepsilon+P f_{q}(\xi),
$$

where

$$
\begin{gathered}
T(\xi)=\left(a d j_{1}(\xi), \ldots, a d j_{\min (N, M)}(\xi)\right), \quad \xi \in \mathbb{R}^{N \times M}, \\
\tau=\sum_{i=1}^{\min (N, M)}\left(\begin{array}{c}
N \\
i
\end{array}\right)\left(\begin{array}{c}
M \\
i
\end{array}\right)
\end{gathered}
$$

(See [4].) Using (3.2), we get

$$
P f_{q}(\xi)>-\varepsilon+P f_{q}(\xi)-\frac{F}{\gamma} w(p-q) \sum_{i=1}^{\tau+1} \lambda_{i}\left(1+\left|\xi_{i}\right|^{p+\gamma}+\left|\xi_{i}\right|^{q+\gamma}\right)
$$

for every $p \in[\alpha, \beta]$. Hence

$$
\limsup _{p \rightarrow q} P f_{p}(\xi) \leqq P f_{q}(\xi) .
$$

Step 2. We suppose in this step that $q>\min (N, M)$ and prove that $\liminf _{p \rightarrow q} P f_{p}(\xi) \geqq P f_{q}(\xi)$. Recalling that $f_{p}$ is lower semicontinuous and verifies (3.1),

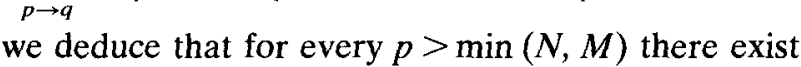

$$
\lambda_{1}^{p}, \ldots, \lambda_{\tau+1}^{p} \in[0,1], \quad \xi_{1}^{p}, \ldots, \xi_{\tau+1}^{p} \in \mathbb{R}^{N \times M} \sum_{i=1}^{\tau+1} \lambda_{i}^{p}=1,
$$

such that

$$
\sum_{i=1}^{\tau+1} \lambda_{i}^{p} T\left(\xi_{i}^{p}\right)=T(\xi) \quad \text { and } \quad \sum_{i=1}^{\tau+1} \lambda_{i}^{p} f_{p}\left(\xi_{i}^{p}\right)=P f_{p}(\xi)
$$

Using the fact that there exists a constant $D>0$ that depends only on $N, M$ such that

$$
\left|T\left(\xi_{i}^{p}\right)\right| \leqq D\left|\xi_{i}^{p}\right|^{\min (N, M)} \quad \text { for every } \quad i=1, \ldots, \tau+1,
$$

adding (3.1) and the fact that $p>\min (N, M)$, we can suppose without restriction 
that the sequence $\left(\left|\xi_{i}^{p}\right|\right)_{p}$ is bounded with respect to $p$. By the fact that $f_{q}$ is lower semicontinuous and $\left(f_{p}\right)_{p}$ verifies (3.2), we find that

$$
\liminf _{p \rightarrow q} P f_{p}(\xi) \geqq P f_{q}(\xi) \text { for every } \quad p>\min (N, M) .
$$

Step 3. We suppose here that $q<\min (N, M), q \neq 2, \ldots, \min (N, M)$ and prove that $\lim \inf P f_{p}(\xi) \geqq P f_{q}(\xi)$. Using Lemma 3.4, knowing that $[p]=[q]$ for $p$ close to $q$ and replacing $T(\xi)$ by $R(\xi)=\left(\operatorname{adj}_{1}(\xi), \ldots, a d j_{[p]}(\xi)\right), \quad \tau$ by $r-1=\sum_{i=1}^{[p]}\left(\begin{array}{c}N \\ i\end{array}\right)\left(\begin{array}{c}M \\ i\end{array}\right), \min (N, M)$ by $[p]$ in the previous step, where $[p]$ is the integer part of $p$, we obtain by the same arguments as those we used in Step 2 that

$$
\liminf _{p \rightarrow q} P f_{p}(\xi) \geqq P f_{q}(\xi) \quad \text { for every } \quad p<\min (N, M), \quad q \neq 2, \ldots, \min (N, M) .
$$

Now (3.9), (3.10) and (3.11) imply that

$$
\lim _{p \rightarrow q} P f_{p}(\xi)=P f_{q}(\xi) \text { for every } q \in[\alpha, \beta], \quad q \neq 2, \ldots, \min (N, M) .
$$

Step 4. We suppose in this step that $q \in\{1, \ldots, \min (N, M)\}$ and prove that $\liminf P f_{p}(\xi)<P f_{q}(\xi)$.

$(\mathrm{i})$ Let $R(\xi)=\left(a d j_{1}(\xi), \ldots, a d j_{q-1}(\xi)\right)$ and $f_{p}(\xi)=|\xi|^{p}+a\left|a d j_{q}(\xi)\right|^{p / q} a>0$. Using the same arguments as in Step 3 , knowing that $[p]=q-1$ for every $p \in(q-1, q)$ and combining with (3.8) of Lemma 3.4, we get that

$$
\liminf _{p \rightarrow q-} P f_{p}(\xi)=\gamma_{q}(\xi)
$$

where

$$
\begin{gathered}
\gamma_{q}(\xi) \equiv \inf \left\{\sum_{i=1}^{s+1} \lambda_{i} f_{q}\left(\xi_{i}\right), \lambda_{i} \in[0,1] \xi_{i} \in \mathbb{R}^{N \times M_{i}}=1, \ldots, s+1,\right. \\
\left.\sum_{i=1}^{s+1} \lambda_{i}=1 \sum_{i=1}^{s+1} \lambda_{i} R\left(\xi_{i}\right)=R(\xi)\right\}, \\
s=\sum_{i=1}^{q-1}\left(\begin{array}{c}
N \\
i
\end{array}\right)\left(\begin{array}{c}
M \\
i
\end{array}\right) .
\end{gathered}
$$

We can see that the infimum is a minimum.

(ii) Applying Lemma 3.4 to $f_{q}$, we get that for suitable $\xi$ and $a>0$ we obtain that $|\xi|^{p}+a\left|a d j_{q}(\xi)\right|>\gamma_{q}(\xi)$. Hence

$$
\liminf _{p \rightarrow q} P f_{p}(\xi)<P f_{q}(\xi) .
$$

This completes the proof of Theorem 3.1. 


\section{Continuity of $Q f_{p}$ with respect to $p$}

We start with the main theorem of this section.

Theorem 4.1. Let $[\alpha, \beta] \subset(1, \infty), F, G, \gamma_{0}>0, C \geqq 1 N, M>1$ be integers and $Q=\left\{x \in \mathbb{R}^{N} ;\left|x_{i}\right| \leqq 3, i=1, \ldots, N\right\}$. Let $w:[0, \infty) \mapsto[0, \infty)$ with $\lim _{t \rightarrow 0} w(t)=w(0)=0$, $\sup \{w(t), t \in[0, \beta]\} \leqq G \quad$ and $\quad f_{p}: \mathbb{R}^{N \times M} \mapsto \mathbb{R} \quad$ lower semicontinuous, $p \in[\alpha, \beta]$, such that:

$$
\begin{gathered}
|\xi|^{p} \leqq f_{p}(\xi) \leqq C\left(1+|\xi|^{p}\right) \quad \text { for every } \quad p \in[\alpha, \beta] \text { and every } \xi \in \mathbb{R}^{N \times M}, \\
\mid f_{p}(\xi)-f_{q}(\xi) \leqq \frac{F}{\gamma} w(p-q)\left(1+|\xi|^{p+\gamma}\right) \text { for every } \gamma \in\left(0, \gamma_{0}\right),
\end{gathered}
$$

every $\xi \in \mathbb{R}^{N \times M}$, and every $p, q \in[\alpha, \beta]$ with $p>q$. Then

$$
Q f_{p}(\xi)=\inf \left\{\frac{1}{|Q|} \int_{Q} f_{p}(\xi+\nabla \phi), \phi \in W_{0}^{1, p}(Q)^{M}\right\}
$$

for every $\xi \in \mathbb{P}^{N \times M}$ and every $p \in[\alpha, \beta]$, and

$$
\lim _{p \rightarrow q} Q f_{p}(\xi)=Q f_{q}(\xi), \quad \text { for every } \xi \in \mathbb{R}^{N \times M} \text { and every } q \in(\alpha, \beta) .
$$

Before proving this theorem, let us begin with some remarks.

Remarks 4.2. (a) The assumption (4.1) says that $f_{p}(\xi)$ behaves at infinity as $|\xi|^{p}$ and can be replaced by $C_{1}\left(|\xi|^{p}-1\right) \leqq f_{p}(\xi) \leqq C\left(1+|\xi|^{p}\right)$.

(b) The assumption (4.2) stands for "continuity" of $f_{p}$ with respect to $p$. This continuity is stronger than the usual continuity and weaker than the uniform continuity.

(c) (4.3) is the result of (4.1) and the characterisation of the quasiconvex envelope. (See [4].)

(d) The idea of the proof of (4.4) is the following: for $p$ fixed, we use (4.3) and approximate $Q f_{p}(\xi)$ by $1 /|Q| \int_{Q} f_{p}\left(\xi+\nabla \phi_{n}\right)$ where $\phi_{n} \in W_{0}^{1, p}(Q)^{N}$. By Gehring's lemma, we deduce that $\left(\phi_{n}\right)_{n}$ is bounded in $W_{\text {loc }}^{1, p+\varepsilon}(Q)^{N}$ for $\varepsilon>0$ independent of $p$. This will lead to (4.4).

Examples 4.3. (a) Let

(2) Let

$$
f_{p}(\xi)=\left\{\begin{array}{lll}
1+|\xi|^{p} & \text { for } & |\xi| \neq 0, \\
0 & \text { for } & |\xi|=0,
\end{array} \quad \xi \in \mathbb{R}^{2 \times 2}\right.
$$

$$
f_{p}(\xi)=|\xi|^{p}+a|\operatorname{det}(\xi)|^{p / 2}, \quad a>2
$$

Using Theorem 4.1 we show that in Examples 4.3 (1) and (2) $Q f_{p}>P f_{p}=C f_{p}$ for $p$ near 2 .

Notation.

- Let $R>0, a \in \mathbb{R}^{N}$. We define: $Q_{R}(a)=\left\{x \in \mathbb{R}^{N},\left|x_{i}-a_{i}\right| \leqq R, i=1, \ldots, N\right\}$, $B_{R}(a)=\left\{x \in \mathbb{R}^{N}, \Sigma_{1 \leqq i \leqq N}\left(x_{i}-a_{i}\right)^{2} \leqq R^{2}\right\}, Q=Q_{3}(0)$;

- for every $x \in \mathbb{R}^{N},|x|_{p}^{p}=\Sigma_{1 \leqq i \leqq N}\left|x_{i}\right|^{p}$ if $1 \leqq p<\infty$ 
and $|x|_{\infty}=\max \left\{\left|x_{i}\right|, i=1, \ldots, N\right\}$

- $\operatorname{dist}(x, y)=|x-y|_{\infty}$ for every $x, y \in \mathbb{R}^{N}$;

- $\oint_{Q} u=\frac{1}{|Q|} \int_{Q} u, u_{R}(a)=\oint_{Q_{R(a)}} u,\|u\|_{r}^{r}=\int_{Q}|u|^{r}$ for every $r \geqq 1$, every $u \in L^{r}(Q)$, every $a \in Q$, and every $R>0$ "small";

- $\mathbb{R}^{N \times N}$ is the set of the $N \times N$ real matrices.

LEMMA 4.4. Let $N \geqq 2$ be an integer, $\beta \in(1, \infty)$ and $\Omega \subset \mathbb{R}^{N}$ a bounded open set with Lipschitz boundary. There exists a constant $C>0$ depending only on $\Omega$ and $\beta$ such that:

$$
\int_{\Omega}|u|^{p} \leqq C\left(\int_{\Omega}|u|^{\mu}\right)^{p / \mu}
$$

for every $p \in[1, \beta], u \in W^{1, p}(\Omega)$ with $\int_{\Omega} u=0$ and for $\mu=\max \{1,(N p) /(N+p)\}$.

Remark 4.5. Lemma 4.4 is exactly Poincaré's inequality and Sobolev's theorem. We want to show that Sobolev's constant $C$ corresponding to the embedding of $W^{1, \mu}(\Omega)$ to $L^{p}(\Omega)$ remains bounded when $p \in K=[1, \beta] \subset \mathbb{R}$. This result is not surprising and is easily proved.

Proof of Lemma 4.4. We divide the proof into two parts.

Part 1. Suppose that $1 \leqq p \leqq N /(N-1)=\tilde{p}$. Using Sobolev's embedding theorem, we find two constants $\bar{C}_{1}, \bar{C}_{2}>0$ depending only on $\Omega$ such that:

$$
\begin{gathered}
\int_{\Omega}|u|^{\tilde{p}} \leqq \bar{C}_{1}\left(\int_{\Omega}(|\nabla u|+|u|)\right)^{\bar{p}} \text { for every } u \in W^{1,1}(\Omega), \\
\int_{\Omega}|u| \leqq \bar{C}_{2}\left(\int_{\Omega}|\nabla u|\right) \text { for every } u \in W^{1,1}(\Omega) \text { verifying } \int_{\Omega} u=0 .
\end{gathered}
$$

(See [3, p. 168] and [12].) By Hölder's inequality,

$$
u \in W^{1, p}(\Omega) \text { implies } \int_{\Omega}|u|^{p} \leqq(1+|\Omega|)\left(\int_{\Omega}|u|^{\tilde{p}}\right)^{p / \tilde{p}} .
$$

From (4.11), (4.12) and (4.13) we find a constant $C_{1}>0$ depending only on $\Omega$ such that $1 \leqq p \leqq \tilde{p}, u \in W^{1, p}(\Omega), \int_{\Omega} u=0$ imply

$$
\int_{\Omega}|u|^{p} \leqq C_{1}\left(\int_{\Omega}|\nabla u|^{\mu}\right)^{p / \mu}
$$

Part 2. We now carry out an induction on $i_{0}$ and suppose that there exist constants $C_{1}, \ldots, C_{i 0}$ such that

$$
p \leqq \frac{N p}{N+i p}<\tilde{p}, \quad i=1, \ldots, i_{0}, \quad u \in W^{1, p}(\Omega) \quad \text { and } \quad \int_{\Omega} u=0
$$

imply that

$$
\int_{\Omega}|u|^{p} \leqq C_{i}\left(\int_{\Omega}|\nabla u|^{\mu}\right)^{p / \mu}
$$


Let

$$
u \in W^{1, p}(\Omega), \quad p \in[1, \beta]
$$

such that

$$
\int_{\Omega} u=0, \quad \frac{N p}{N+\left(i_{0}+1\right) p}<\tilde{p} \leqq \frac{N p}{N+i_{0} p} \quad \text { and } \quad \mu_{1}=\max \left\{1, \frac{N p}{N+\left(i_{0}+1\right) p}\right\} .
$$

We find: $\mu_{1}=(N p) /\left(N+\left(i_{0}+1\right) p\right)<\tilde{p}, \mu=(N p) /(N+p)$. By our induction hypothesis, we get:

$$
\int_{\Omega}|u|^{\mu} \leqq C_{i_{0}}\left(\int_{\Omega}|\nabla u|^{\mu_{1}}\right)^{\mu / \mu_{1}}
$$

Let $P: W^{1, p}(\Omega) \mapsto W^{1, p}\left(\mathbb{R}^{N}\right)$ be the extension operator. (See [3, pp. 158-162].) We obtain

$$
\|u\|_{p} \leqq\|P u\|_{L^{p}\left(\mathbb{R}^{N}\right)} \leqq \beta\|\nabla P u\|_{L^{\mu}\left(\mathbb{R}^{N)}\right.} \leqq \beta \bar{C}_{3}\left(\|u\|_{\mu}+\|\nabla u\|_{\mu}\right),
$$

with $\bar{C}_{3}$ depending only on $\Omega$ and $\|u\|_{\mu}$ denoting $\|u\|_{L^{\mu}(Q)}$. Using Hölder's inequality in (4.15) and adding (4.16), we can conclude that there exists a constant $C_{i_{0}+1}>0$ depending only on $\Omega, \beta, i_{0}$ such that: $\int_{\Omega}|u|^{p} \leqq C_{i_{0}+1}\left(\int_{\Omega}|\nabla u|^{\mu}\right)^{p / \mu}$. Assuming that $C=\max \left\{C_{1}, \ldots, C_{i}\right\}$ with $(N \beta) /(N+i \beta)<\tilde{p}=N /(N-1)$, we obtain the existence of a constant $C>0$ depending only on $\Omega$ and $\beta$ such that: $p \in[1, \beta], u \in W^{1, p}(\Omega)$ and $\int_{\Omega} u=0$ imply $\xi_{\Omega}|u|^{p} \leqq C\left(\int_{\Omega}|\nabla u|^{\mu}\right)^{p / \mu}$.

Lemma 4.6. let $a \in \mathbb{R}^{N}, R>0$ be real, $v>1$ be an integer. Assume that $A_{0}=Q_{R}(a), A_{i}=\left\{x \in \mathbb{R}^{N}: \operatorname{dist}\left(x, Q_{R}(a)\right)<(i r) / v\right\}, i=1, \ldots, v$. Then there exist $\phi_{i} \in C_{0}^{1}\left(A_{i}\right), i=1, \ldots, v$ such that

$$
0 \leqq \phi_{i}(x) \leqq 1, \quad x \in A_{i}, \quad \phi_{i}(x)=1, x \in A_{i-1}, \quad\left|\nabla \phi_{i}(x)\right| \leqq \frac{v+1}{R}, \quad x \in A_{i} .
$$

The proof of Lemma 4.6 is elementary.

Lemma 4.7. Let $b, q>1, r>q, N>0$ an integer, $\theta<1 /\left(a_{1}(q)\right)=1 /\left(30^{N}(q-1)\right)$ $\left((q-1 /(5 q))^{q}\right.$ and $g, h: Q=\left\{x \in \mathbb{R}^{N},\left|x_{i}\right| \leqq 3, i=1, \ldots, N\right\} \mapsto[0, \infty)$ be two functions such that $g \in L^{q}(Q)$ and $h \in L^{r}(Q)$. Suppose that for every $x_{0} \in Q$, every $0<R<\frac{1}{2} \operatorname{dist}\left(x_{0}, \partial Q\right)$

$$
\oint_{Q_{R}\left(x_{0}\right)} g^{q} \leqq b\left\{\left(\oint_{Q_{2 R}\left(x_{0}\right)} g\right)^{q}+\oint_{Q_{2 R}\left(x_{0}\right)} h^{q}\right\}+\theta \oint_{Q_{2 R}\left(x_{0}\right)} g^{q}
$$

Then:

$$
\int_{D_{k}} g^{t} \leqq C(t, q)(3)^{N t / q}\left(2^{k}\right)^{N t / q}\|g\|_{q}^{t}\left[\int_{Q} g^{q}+\int_{Q} h^{t}\right] \text { for every } t \in[q, q+\varepsilon)
$$


where

$$
\begin{gathered}
\varepsilon=\min \left\{r-q, \frac{q-1}{a-1}\right\}, \quad a \equiv a(q, b, \theta)=\frac{a_{1}(q)+a_{2}(q)}{1-\theta a_{1}(q)} b, \quad a_{2}(q)=2^{N}\left(\frac{5 q}{q-1}\right)^{q-1}, \\
C_{-1}=\left\{x \in \mathbb{R}^{N},\left|x_{i}\right| \leqq 1, i=1, \ldots, N\right\}, \\
C_{k}=\left\{x \in \mathbb{R}^{N}, \frac{1}{2^{k}}<\operatorname{dist}(x, \partial Q) \leqq \frac{1}{2^{k-1}}\right\} \quad D_{k}=\bigcup_{i=-1}^{i=k} C_{k} \quad k=-1,0,1, \ldots
\end{gathered}
$$

Proof. The proof of the above lemma has been given in [7] by Giaquinta and Modica. It is based on Gehring's lemma.

As an illustration, we give the following lemma in the case $N=M$. For the general case $(N, M>1)$ see [10].

Lemma 4.8. Let $p \in[\alpha, \beta] \subset(1, \infty), C \geqq 1, N \geqq 2,0 \leqq \eta \leqq 1 f: \mathbb{R}^{N \times N} \mapsto \mathbb{R}$ be a Borel measurable function, $Q=\left\{x \in \mathbb{R}^{N},\left|x_{i}\right| \leqq 3, \quad i=1, \ldots, N\right\}, \quad u \in W^{1, p}(Q)^{N}$ such that:

$$
\begin{gathered}
|\xi|^{p} \leqq f(\xi) \leqq C\left(1+|\xi|^{p}\right) \quad \text { for every } \quad \xi \in \mathbb{R}^{N \times N}, \\
F(A, u) \leqq F(A, v)+\eta \int_{A}|\nabla u-\nabla v|,
\end{gathered}
$$

for every $A \Subset Q$ an open set and every $v \in u+W_{0}^{1, p}(A)^{N}$ with $F(A, v)=\int_{A} f(\nabla v)$. Then

$$
\oint_{Q_{R}\left(x_{0}\right)}|\nabla u|^{p} \leqq b\left\{\left(\oint_{Q_{2 R}\left(x_{0}\right)}|\nabla u|^{\mu}\right)^{p / \mu}+\oint_{Q_{2 R}\left(x_{0}\right)} h^{\rho / \mu}\right\}+\frac{2^{p+1} C}{v} \oint_{Q_{2 R}\left(x_{0}\right)}|\nabla u|^{p},
$$

for every $x_{0} \in Q$, every $0<R<\frac{1}{2} \operatorname{dist}\left(x_{0}, \partial Q\right)$ and every $v>1$ integer, where $\mu=\max \{1,(N p) /(N p+1)\}, h=\left(3+\|\nabla u\|_{2}\right)^{\mu / p}, b=\left\{2^{p+1} D(p)(v+1)^{p}+1\right\} \times$ $\left\{2^{p-1+N} C+1\right\}$ and $D(p)$ is defined (see Lemma 4.4) by $\int_{\Omega}|u|^{p} \leqq D(p)\left(\int_{Q} \mid \nabla u \|^{\mu}\right)^{p / \mu}$ for every $u \in W^{1, p}(Q)$ such that $\int_{Q} u=0$. Further, there exist two constants $m_{3}, E>0$ depending only on $\alpha, \beta, C$ such that:

$$
\int_{D_{k}}|\nabla u|^{s} \leqq E(3)^{N s / p}\left(2^{k}\right)^{N s / p}\|\nabla u\|_{p}^{s}\left[\int_{Q}|\nabla u|^{p}+\int_{Q}(3+|\nabla u|)^{s / p}\right],
$$

for every $k=-1,0,1, \ldots$ and every $s \in\left[p, p+m_{3}\right]$.

Proof. The proof of (4.25) has been given in [10] by Marcellini and Sbordone. It is easy to reproduce their proof assuming that $f$ satisfies (4.23), $f$ depends only on $\nabla u$ and $f$ is Borel measurable. We now prove (4.26). Using (4.25), we get:

$$
\oint_{Q_{R}\left(x_{0}\right)}|\nabla u|^{p} \leqq b\left\{\left(\oint_{Q_{2 R}\left(x_{0}\right)}|\nabla u|^{\mu}\right)^{p / \mu}+\oint_{Q_{2 R}\left(x_{0}\right)} h^{p / \mu}\right\}+\frac{2^{p+1} C}{v} \oint_{Q_{2 R}\left(x_{0}\right)}|\nabla u|^{p},
$$


for every $x_{0} \in Q$, every $0<R<\frac{1}{2} \operatorname{dist}\left(x_{0}, \partial Q\right)$ and every $v>1$ integer. Let

We obtain

$$
A_{1}=\sup \left\{a_{1}(q), q \in(1, \beta]\right\}, \quad v>2^{\beta+2} C A_{1} .
$$

$$
\theta<\frac{1}{2 A_{1}}, \quad 0<a(q, b, \theta) \leqq 4 A_{1} b
$$

Assuming that

$$
\begin{gathered}
g=|\nabla u|^{\mu}, \quad q=\frac{p}{\mu}, \quad t=\frac{s}{\mu} \\
m_{3}=\frac{1}{2} \min \left\{(\alpha-1), \frac{\alpha-1}{A_{1}-1}, \frac{\alpha^{2}}{N+\beta}\right\} \\
E=\sup \left\{\left|c\left(\frac{s}{\mu}, \frac{p}{\mu}\right)\right|+1, p \in[\alpha, \beta], s \in\left[p, p+m_{3}\right]\right\}
\end{gathered}
$$

and using Lemma 4.7, we find (4.26).

Remark 4.9. One can see that

$$
s \leqq p^{2} \quad \text { for every } s \in\left[p, p+m_{3}\right)
$$

and, by Hölder's inequality, (4.26) implies:

$$
\int_{D_{k}}|\nabla u|^{s} \leqq E 2^{\beta}|Q|\left(3.2^{k}\right)^{N s / p}\|\nabla u\|_{p}^{s}\left[1+3^{\beta}+2\|\nabla u\|_{p}^{\beta}\right]\left[1+\|\nabla u\|_{p}^{\beta}\right] .
$$

We now proceed with the proof of Theorem 4.1.

Proof of Theorem 4.1. To illustrate, we give the proof in the case $N=M$. Fix $\xi \in \mathbb{R}^{N \times N}$.

Part 1. The Proof of (4.3) is elementary.

Part 2. We prove (4.4). We decompose the proof into six steps.

Step 1. Let

$$
\begin{aligned}
& V=W_{0}^{1,1}(Q)^{N}
\end{aligned}
$$

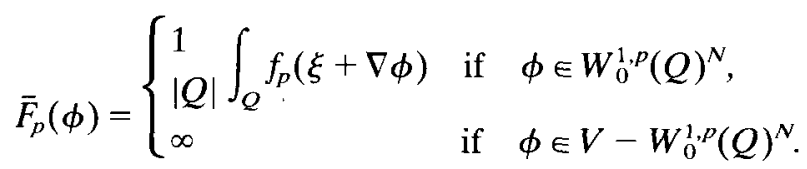

Here, we show that there exists a sequence $\left(\phi_{n}^{p}\right)_{n} \in V$ such that:

$$
\int_{D_{k}}\left|\xi+\nabla \phi_{n}^{p}\right|^{s} \leqq J 2^{N k \beta}\left[1+\left\|\xi+\nabla \phi_{n}^{p}\right\|_{p}^{2 \beta}\right] \text { and } \lim _{n \rightarrow \infty} \bar{F}_{p}\left(\phi_{n}^{p}\right)=Q f_{p}(\xi)
$$

for every $s \in\left[p, p+m_{3}\right]$ and every $k=-1,0,1, \ldots$, where $D_{k}, m_{3}$ are defined in Lemma 4.7 , Lemma 4.8 and $J$ is a constant depending only on $Q, \beta$. Observe that

$$
f_{p} \text { is lower semicontinuous } \Rightarrow \bar{F}_{p} \text { is lower semicontinuous. }
$$


By (4.3) and (4.34) we deduce that there exists a sequence

$$
\left(\psi_{n}^{p}\right)_{n} \in V \quad \text { with } \quad F_{p}\left(\psi_{n)}^{p} \leqq \inf \left\{\bar{F}_{p}(\phi), \phi \in V\right\}+\frac{1}{n}=Q f_{p}(\xi)+\frac{1}{n} .\right.
$$

Using the variational principle of Ekeland (see [5]), we obtain a sequence

$$
\left(\phi_{n}^{p}\right)_{n} \in V \quad \text { such that } \quad \bar{F}_{p}\left(\phi_{n}^{p}\right) \leqq \bar{F}_{p}\left(\psi_{n}^{p}\right) \text { with } \int_{Q}\left|\nabla \psi_{n}^{p}-\nabla \phi_{n}^{p}\right| \leqq 1
$$

and

$$
\bar{F}_{p}\left(\phi_{n}^{p}\right) \leqq \bar{F}_{p}(\phi)+\frac{1}{n} \int_{Q}\left|\nabla \phi-\nabla \phi_{n}^{p}\right| \quad \text { for every } \quad \phi \in V .
$$

Further, for every $A$ an open set, $A \Subset Q$ and every $\phi \in \phi_{n}^{p}+W_{0}^{1, p}(A)^{N}$, we obtain

where

$$
\bar{F}_{p}\left(A, \phi_{n}^{p}\right) \leqq \bar{F}_{p}(A, \phi)+\frac{1}{n} \int_{Q}\left|\nabla \phi-\nabla \phi_{n}^{p}\right|
$$

$$
\bar{F}_{p}(A, \phi)=\frac{1}{|Q|} \int_{A} f_{p}(\xi+\nabla \phi) \quad \text { if } \quad \phi \in W^{1, p}(A)^{N} .
$$

In the next four steps, we suppose that $q \in(\alpha, \beta)$ is a fixed number.

Step 2. Let $n$ be a fixed integer. We show that for every sequence $S \subset[\alpha, q]$ there exists a subset $\bar{S} \subset S$, a subsequence $\left(\phi_{n}^{r}\right)_{r \in \bar{S}}$ and a $\phi_{n} \in W_{0}^{1, q}(Q)^{N}$ such that:

$$
\phi_{n}^{r} \underset{r \rightarrow q}{\stackrel{\text { weakly }}{\longrightarrow}} \phi_{n} W^{1, p}(Q)^{N} \quad \text { for every } p \in \bar{S} \text {. }
$$

By (4.2), (4.35) and (4.36), for every $p \in S$ such that $p$ is near $q$, we find that:

$$
\frac{1}{|Q|} \int_{Q}\left|\xi+\nabla \phi_{n}^{p}\right|^{p} \leqq f_{p}(\xi)+1 \leqq f_{q}(\xi)+1+\frac{F G}{\gamma_{0}}\left(1+|\xi|^{q+\gamma_{0}}\right)=H(\xi) .
$$

We choose with respect to $p$ a subsequence in the following way. First, we fix $p_{1}^{1} \in S$. Using Hölder's inequality in (4.41), we deduce that there there exists a sequence $p_{1}^{1}<p_{2}^{1}<p_{3}^{1}<, \ldots$, in $S$ such that

$$
\phi_{n}^{p^{l}} \stackrel{\text { weakly }}{\longrightarrow} l_{n}^{p_{1}^{1}} W^{1, p 1}(Q)^{N} \text { and } p_{i}^{1} \underset{i \rightarrow \infty}{\longrightarrow} q .
$$

Then assume that $p_{1}^{2}=p_{1}^{1}, p_{2}^{2}=p_{2}^{1}$. Using Hölder's inequality again in (4.41), we deduce that there exists $p_{1}^{2}<p_{2}^{2}<p_{3}^{2}<, \ldots$, in $S$ such that

$$
\phi_{n}^{p_{i}^{2}} \underset{i \rightarrow \infty}{\text { weakly }} l_{n}^{p^{2}} W^{1, p_{2}^{2}}(Q)^{N}, p_{i}^{2} \underset{i \rightarrow \infty}{\longrightarrow} q \text { and } l_{n}^{p^{1}}=l_{n}^{p_{2}^{2}}
$$

Now suppose that we have found the numbers $p_{1}^{1}<p_{2}^{2}<\ldots<p_{k}^{k}$ and an increasing subsequence $\left(p_{i}^{k}\right)_{i \in \mathbb{N}}$ such that

$$
\phi_{n}^{p_{i}^{k} \text { weakly }} \underset{i \rightarrow \infty}{\longrightarrow} l_{n}^{p_{k}^{k}} W^{1, p !}(Q)^{N}, \ldots, W^{1, p_{k}^{k}}(Q)^{N}
$$

Assume that $p_{1}^{k+1}=p_{1}^{k}, \ldots, p_{k+1}^{k+1}=p_{k+1}^{k}$. Using Hölder's inequality again in 
(4.41), we can obtain an increasing subsequence $\left(p_{i}^{k+1}\right)_{i \geqq k+2}$ from $\left(p_{i}^{k}\right)_{i \geqq k+2}$ such that

$$
\phi_{n}^{p_{k}^{k+1}} \stackrel{\text { weakly }}{\longrightarrow} l_{n}^{p_{k+1}^{k+1}} W^{1, p_{k+1}^{k+1}}(Q)^{N} \quad \text { and } \quad l_{n}^{p_{k}^{k}}=l_{n}^{k_{k+1}^{k+1}} .
$$

Assume that $\phi_{n}=l_{n}^{k+1}$ and $\bar{S}=\left\{p_{k}^{k}, k \in \mathbb{N}\right\}$. Using (4.41), it is easy to deduce that $\phi_{n} \in W_{0}^{1, q}(Q)^{N}$ and

$$
\phi_{n}^{r} \stackrel{\text { weakly }}{\longrightarrow} \phi_{n} W^{1, p}(Q)^{N} \quad \text { for every } \quad p \in \bar{S} .
$$

Step 3. We show that

$$
\liminf _{p \rightarrow q^{-}} Q f_{p}(\xi) \geqq Q f_{q}(\xi),
$$

where $\liminf _{p \rightarrow q-} Q f_{p}(\xi)$ is defined by $\liminf _{p \rightarrow q, p<q} Q f_{p}(\xi)$. To show (4.45), we suppose that

$$
\liminf _{p \rightarrow q^{-}} Q f_{p}(\xi)<Q f_{q}(\xi)
$$

and we get a contradiction. Now (4.46) implies that there exists a sequence $\left(p_{i}\right)_{i} \subset[\alpha, q]$ such that $\lim _{i \rightarrow \infty} Q f_{p_{i}}(\xi)<Q f_{q}(\xi)$. Let $m_{3}$ and $D_{k}$ be defined as in Lemma 4.7 and assume that $\gamma=\min \left\{\gamma_{0}, \frac{m_{3}}{8}\right\}$. By (4.35) and (4.36), we get $Q f_{p_{i}}(\xi) \geqq \frac{1}{|Q|} \int_{Q} f_{p_{i}}\left(\xi+\nabla \phi_{n}^{p_{i}}\right)+\frac{1}{n}$. Assuming that $|p-q|<m_{3}\left(m_{3}\right.$ is defined in Lemma 4.8) and using (4.2) and (4.33), we deduce that for every $k=-1,0,1, \ldots$

$$
Q f_{p_{i}}(\xi) \geqq \frac{1}{|Q|} \int_{D_{k}} f_{q}\left(\xi+\nabla \phi_{i}^{p} n\right)-\frac{1}{n}-\frac{F G}{\gamma|Q|} w\left(p_{i}-q\right) J(\beta, k, \gamma, \xi)
$$

and $\lim _{i \rightarrow \infty} Q f_{p_{i}}(\xi) \geqq \liminf _{i \rightarrow \infty} 1 /|Q| \int_{D_{k}} f_{q}\left(\xi+\nabla \phi_{n}^{p_{i}}\right)-1 / n$. Using the fact that $Q f_{q}$ is quasiconvex (see [4]), we obtain

$$
\lim _{i \rightarrow \infty} Q f_{p_{i}}(\xi) \geqq \frac{1}{|Q|} \int_{D_{k}} Q f_{q}\left(\xi+\nabla \phi_{n}\right)-\frac{1}{n} .
$$

Recalling that $\phi_{n} \in W_{0}^{1, q}(Q)^{N}$ and that (4.47) is true for every $k=-1,0,1, \ldots$, we conclude that

$$
\lim _{i \rightarrow \infty} Q f_{p_{i}}(\xi) \geqq Q f_{q}(\xi) .
$$

Therefore (4.46) leads to a contradiction. This implies that (4.46) is false and so (4.45) is proved.

Step 4. We show that

$$
\liminf _{p \rightarrow q^{-}} Q f_{p}(\xi) \leqq Q f_{q}(\xi) .
$$

By (4.35) and (4.36), we get $Q f_{q}(\xi) \geqq Q f_{p}(\xi)-\left(1 /|Q| \int_{Q} u_{n}^{p}\right)-(1 / n)$, where 
$u_{n}^{p}=\left|f_{q}\left(\xi+\nabla \phi_{n}^{q}\right)-f_{p}\left(\xi+\nabla \phi_{n}^{q}\right)\right|$. Using a subsequence of $\left(u_{n}^{p}\right)$ with respect to $p$, we obtain that

$$
u_{n}^{p} \underset{p \rightarrow q-}{\longrightarrow} 0 L^{1}(Q)
$$

Thus for every $n \in \mathbb{N}, Q f_{q}(\xi) \geqq \liminf _{p \rightarrow q^{-}} Q f_{p}(\xi)-(1 / n)$. This leads to (4.49).

Step 5. We show that

$$
\liminf _{p \rightarrow q^{+}} Q f_{p}(\xi) \geqq Q f_{1}(\xi),
$$

where $\liminf _{p \rightarrow q+} Q f_{p}(\xi)$ is defined by $\liminf _{p \rightarrow q, p>q} Q f_{p}(\xi)$. By (4.35) and (4.36) we find that $Q f_{p}(\xi) \geqq 1 /|Q| \int_{Q} f_{p}\left(\xi+\nabla \phi_{n}^{p}\right)-(1 / n)$ and $\left(\phi_{n}^{p}\right)_{p}$ is bounded in $W^{1, q}(Q)^{N}$. Using a subsequence of $\left(\phi_{n}^{p}\right)_{p}$ with respect to $p$, we deduce that

$$
\phi_{n}^{p} \underset{p \rightarrow q+}{\stackrel{\text { weakly }}{\longrightarrow}} \psi_{n} W^{1, q}(Q)^{N} \text {. }
$$

We proceed as in Steps 3 and 4 to conclude that $\liminf _{p \rightarrow q^{+}} Q f_{p}(\xi) \geqq Q f_{q}(\xi)$, which proves (4.50).

Step 6. It is very easy to prove that

$$
\limsup _{p \rightarrow q} Q f_{p}(\xi) \leqq Q f_{q}(\xi)
$$

In conclusion, (4.45), (4.49), (4.50) and (4.51) imply that:

Step 6. It is very easy to prove that

$$
\limsup _{p \rightarrow q} Q f_{p}(\xi) \leqq Q f_{q}(\xi)
$$

In conclusion, (4.45), (4.49), (4.50) and (4.51) imply that:

$$
\lim _{p \rightarrow q} Q f_{p}(\xi)=Q f_{q}(\xi)
$$

and Theorem 4.1 is completely proved.

We now use Theorem 4.1 to find some quasiconvex functions $f: \mathbb{R}^{N \times N} \rightarrow \mathbb{R}$ which are not polyconvex. We study such functions below.

EXAMPLE 4.10 . Let $C, F>0, \quad \beta>2, \quad f_{p}(\xi)=|\xi|^{p}+a h_{p}(\operatorname{det}(\xi)), \quad \xi \in \mathbb{R}^{2 \times 2}$, $p \in[\alpha, \beta] \subset(1, \infty)$ and $a \geqq 0$ such that $h_{p}(x)$ behaves as $|x|^{p / 2}$. This means that:

$h_{p}: \mathbb{R} \rightarrow \mathbb{R}$ is lower semicontinuous;

$$
h_{p}(x) \leqq h_{q}(x) \text { for } p<q \text { and }|x| \geqq 1 ;
$$

$|x|^{p / 2} \leqq h_{p}(x) \leqq C\left(1+|x|^{p / 2}\right)$ for every $x \in \mathbb{R}$ and every $p \in[\alpha, \beta]$;

$$
h_{2} \text { is convex and } h_{2}(1)>h_{2}(0) \text {; }
$$

$$
\left|h_{p}(x)-h_{q}(x)\right| \leqq \frac{F}{\gamma}|p-q|\left(1+|x|^{(q / 2)+\gamma}\right) \quad \text { for every } \quad p<q \in[\alpha, \beta]
$$

and every $\gamma \in\left[0, \frac{1}{2}\right]$. Then for every $\alpha>\frac{2}{h_{2}(1)-h_{2}(0)}$ there exists a $p_{0} \in(1,2)$ such that

$$
Q f_{p}>P f_{p} \text { for } p_{0}<p<2 \text {. }
$$


Proof. (We note that $h_{p}(x)=|x|^{p / 2}$ satisfies the hypotheses above.) First, $h_{2}$ convex and $a \geqq 0$ imply that $f_{2}$ is polyconvex; $a>\frac{2}{h_{2}(1)-h_{2}(0)}$ implies that $f_{2}$ is not convex. Using (4.53) and (4.56), we find that:

$$
\limsup _{p \rightarrow 2} C f_{p} \leqq C f_{2} \text {. }
$$

By Theorem 4.1 and (4.56) we find that

$$
\liminf _{p \rightarrow 2} Q f_{p}=Q f_{2}>C f_{2} \geqq \limsup _{p \rightarrow 2} C f_{p}=\limsup _{p \rightarrow 2} P f_{p} .
$$

Knowing that $P f_{2}=f_{2}$ and $P f_{p}=C f_{p}$ for every $p \in(1,2)$, we conclude that there exists a $p_{0} \in(1,2)$ such that

$$
Q f_{p}>P f_{p} \text { for } p \in\left(p_{0}, 2\right) \text {. }
$$

EXAMPLE 4.11. A particular case of this example has been studied in [8] by Kohn. Let $N>1$ be an integer, $\beta>2, F, \gamma_{0}, d>0, M \geqq 1, I$ the identity matrix of $\mathbb{R}^{N \times N}, p \in[\alpha, \beta] \subset(1, \infty)$ and

$$
f_{p}(\xi)=\min \left(|\xi+I|^{p},|\xi-I|^{p}\right)+h_{p}(|\operatorname{det}(\xi)|-1), \quad \xi \in \mathbb{R}^{N \times N}, \quad\left(h_{p} \equiv 0 \text { in }[8]\right),
$$

with $h_{p}(x)$ behaving as $|x|^{p / N}$. This means that:

$$
\begin{gathered}
h_{p}: \mathbb{R} \rightarrow \mathbb{R} \text { is lower semicontinuous; } \\
\left.h_{p}(0)=0, \sup \left\{\left|h_{p}(x)\right| ;|x| \leqq M\right\}<M\right\}<F \text { for every } p \in[\alpha, \beta] \\
h_{p}(x) \leqq h_{q}(x) \text { for } p<q \text { and }|x| \geqq M ; \\
0 \leqq h_{p}(x) \leqq d\left(1+|x|^{p / N}\right) \text { for every } x \in \mathbb{R} \text { and every } p \in[\alpha, \beta] ; \\
\left|h_{p}(x)-h_{q}(x)\right| \leqq \frac{F}{\gamma}|p-q|\left(1+|x|^{(q / N)+\gamma}\right) \text { for every } p<q \in[\alpha, \beta]
\end{gathered}
$$

and every $\gamma \in\left(0, \gamma_{0}\right]$. Then there exists a $p_{0} \in(1,2)$ such that

$$
Q f_{p}>P f_{p} \text { and } p \in\left(p_{0}, 2\right)
$$

Proof. (We first note that $h_{p}(x)=|x|^{p / N}$ satisfies the hypotheses above.) We find that $P F_{2}(\xi)=0 \Rightarrow \xi=I,-I$ and $C f_{2}(t I)=0$ for every $t \in[0,1]$. Therefore

$$
P f_{2}>C f_{2} \text {. }
$$

Assuming that $g_{p}(\xi)=f_{p}(\xi)+N^{\beta / 2}$, we find that $g$ satisfies the hypotheses of Theorem 4.1. Thus $\liminf _{p \rightarrow 2} Q f_{p}=Q f_{2}$. (4.59), (4.61) and (4.60) imply that $\limsup _{p \rightarrow 2} C f_{p} \leqq C f_{2}$. We then conclude that:

$$
\underset{p \rightarrow 2}{\liminf } Q f_{p}=Q f_{2} \geqq P f_{2}>C f_{2} \geqq \limsup _{p \rightarrow 2} C f_{p} .
$$

Therefore there exists a $p_{0} \in(1,2)$ such that

$$
Q f_{p}>P f_{p} \text { for every } p \in\left(p_{0}, 2\right) \text {. }
$$




\section{Continuity of $R f_{p}$ with respect to $p$}

We first start with the main theorem of this section.

Theorem 5.1. Let $[\alpha, \beta] \subset(1, \infty), F, G, K, \gamma_{0}>0, C \geqq 1$ and $N, M>1$ be two integers. Let $w:[0, \infty) \mapsto[0, \infty)$ with $\lim _{t \rightarrow 0} w(t)=w(0)=0, \sup \{w(t), t \in[0, \beta]\} \leqq G$ and $f_{p}: \mathbb{R}^{N \times M} \mapsto \mathbb{R}$ lower semicontinuous, $p \in[\alpha, \beta]$, such that:

$$
\begin{gathered}
|\xi|^{p} \leqq f_{p}(\xi) \leqq C\left(1+|\xi|^{p}\right) \quad \text { for every } \quad p \in[\alpha, \beta] \text { and every } \xi \in \mathbb{R}^{N \times M} \\
\mid f_{p}(\xi)-f_{q}(\xi) \leqq \frac{F}{\gamma} w(p-q)\left(1+|\xi|^{p+\gamma}\right) \quad \text { for every } \gamma \in\left(0, \gamma_{0}\right)
\end{gathered}
$$

every $\xi \in \mathbb{R}^{N \times M}$, and every $p, q \in[\alpha, \beta]$ with $p>q$;

Then

$$
f_{p}(\xi)=R f_{p}(\xi) \text { for every }|\xi| \geqq K \text {. }
$$

$\lim _{p \rightarrow q} R f_{p}(\xi)=R f_{q}(\xi), \quad$ for every $\xi \in \mathbb{R}^{N \times M}$ and every $q \in(\alpha, \beta)$.

Before proving this theorem, let us begin with some remarks.

Remarks 5.2. (a) In general, we have $\liminf _{p \rightarrow 1^{-}} R f_{p}<R f_{1}$. Indeed, if $f_{p}(\xi)=|\xi|^{p}, \xi \in \mathbb{R}^{N \times M}$ then $0 \equiv \liminf _{p \rightarrow 1^{-}} R f_{p}<R f_{1}=f_{1}$.

(b) Theorem 5.1 is still true if we replace the condition (5.1) by $a+b|\xi|^{p} \leqq f_{p}(\xi) \leqq C\left(1+|\xi|^{p}\right)$, where $a \in \mathbb{R}$ and $b>0$ are two constants.

(c) We do not need to use (5.3) to prove that $\limsup _{p \rightarrow q} R f_{p} \leqq R f_{q}$ for $q \in[\alpha, \beta]$. The most difficult part in this case is to prove that $\liminf _{p \rightarrow q} R f_{p} \geqq R f_{q}$. We were unable to prove this inequality without assuming (5.3).

(d) If we keep only hypotheses (5.1) and (5.2), we can show that

$$
\lim _{p \rightarrow q} R_{k} f_{p}=R_{k} f_{q} \quad \text { for every } k \in \mathbb{N} \text {, and every } q \in[\alpha, \beta] .
$$

Where $R_{0} f=f, R_{k+1} f(\xi)=\inf \left\{t R_{k} f(\eta)-(1-t) R_{k} f(\mu), t \in(0,1), \eta, \mu \in \mathbb{R}^{N \times M}\right.$, $\operatorname{rank}(\eta-\mu) \leqq 1, \xi=t \eta+(1-t) \mu\}$. One knows that $\lim _{k \rightarrow \infty} R_{k} f=R f$. (See $[4,9]$.)

EXAMPLE 5.3. Let

$$
f_{p}(\xi)=\left\{\begin{array}{lll}
1+\mid \xi^{p} & \text { for } & |\xi| \neq 0, \\
0 & \text { for } & |\xi|=0,
\end{array} \quad \xi \in \mathbb{R}^{N \times M} .\right.
$$

Knowing that $C f_{p}(\xi)=1+|\xi|^{p}$ if $|\xi| \geqq(1 /(p-1))^{1 / p}$, we find that $\left(f_{p}\right)_{p}$ verifies (5.1), (5.2) and (5.3). Hence Theorem 5.1 leads to $\lim _{p \rightarrow q} R f_{p}=R f_{q}$, for every $q>1$.

To prove Theorem 5.1, let us now begin with the following lemma: 
Lemma 5.4. Let $N, M \geqq 1$ be two integers, $C, K>0$ two real constants and $f: \mathbb{R}^{N \times M} \mapsto \mathbb{R}$ lower semicontinuous such that:

$$
\begin{gathered}
|\xi|^{p} \leqq f_{p}(\xi) \leqq C\left(1+|\xi|^{p}\right) \quad \text { for every } \xi \in \mathbb{R}^{N \times M} ; \\
R f(\xi)=f(\xi) \text { for every }|\xi| \geqq K .
\end{gathered}
$$

Then, for every $\xi \in \mathbb{R}^{N \times M}$, such that $|\xi| \leqq K$, there exist $t \in[0,1], \xi_{1}, \xi_{2} \in \mathbb{R}^{N \times M}$ verifying

$$
\begin{aligned}
\operatorname{rang}\left(\xi_{1}-\xi_{2}\right) \leqq 1, \quad \xi=t \xi_{1}+(1-t) \xi_{2}, \quad R_{1} f(\xi) & =t R_{1} f\left(\xi_{1}\right) \\
& +(1-t) R_{1} f\left(\xi_{2}\right),\left|\xi_{1}\right|,\left|\xi_{2}\right| \leqq K .
\end{aligned}
$$

Proof. The proof of Lemma 5.4 is left to the reader.

We now prove Theorem 5.1.

Proof of Theorem 5.1. Let $\xi \in \mathbb{R}^{N \times M}$ and $q \in[\alpha, \beta]$. Using the same arguments as in Step 1 of the proof of Theorem 3.1, we prove that $\limsup _{p \rightarrow q} R f_{p}(\xi) \leqq R f_{q}(\xi)$.

Let us now prove that $\lim \inf R f_{p}(\xi) \geqq R f_{q}(\xi)$. Using (5.1), the result is obvious if $|\xi| \geqq K$. If $|\xi| \leqq K$, by (5.1), (5.2), (5.3) and Lemma 5.4, we get that, for every $k \in \mathbb{N}$, for every $p \in[\alpha, \beta]$, there exist $\lambda_{i}^{p} \in(0,1), \xi_{i}^{p} \in \mathbb{R}^{N \times M}, i=1, \ldots, 2^{k}$ such that

$$
\left|\xi_{i}^{p}\right| \leqq K, \quad i=1, \ldots, 2^{k}, \quad R f_{p}(\xi)=\sum_{i=1}^{2^{k}} \lambda_{i}^{p} f_{p}\left(\xi_{i}^{p}\right) \quad \text { and } \quad R f_{q}(\xi) \leqq \sum_{i=1}^{2^{k}} \lambda_{i}^{p} f_{q}\left(\xi_{i}^{p}\right)
$$

We deduce from the previous relations that

$$
R f_{p}(\xi) \geqq R f_{q}(\xi)-\frac{F}{\gamma} w(p-q)\left(1+M^{p+\gamma}+M^{p+\gamma}\right),
$$

and so

$$
\liminf _{p \rightarrow q} R f_{p}(\xi) \geqq R f_{q}(\xi) .
$$

Hence Theorem 5.1 is proved.

\section{Examples of $f$ such that $f(\xi)=g(|\xi|)$ and $P f \neq Q f$}

THEOREM 6.1. Let $f: \mathbb{R}^{2 \times 2} \mapsto \mathbb{R}$ be a Borel measurable function, $a, b, c, \alpha>0$, $d \in \mathbb{R}$ and $q \in(1,2)$ such that

$$
\begin{gathered}
a|\xi|+f(0) \leqq f(\xi) \quad \text { for every } \quad \xi \in \mathbb{R}^{2 \times 2}, \quad \text { with equality for } \xi^{\prime}=\frac{\alpha}{\sqrt{2}}\left(\begin{array}{ll}
1 & 0 \\
0 & 1
\end{array}\right) \\
b|\xi|^{q}+d \leqq f(\xi) \leqq c\left(1+|\xi|^{q}\right) \text { for every } \xi \in \mathbb{R}^{2 \times 2}
\end{gathered}
$$

there exists $t_{0} \in(0,1)$ for which $f\left(\xi^{*}\right) \neq a\left|\xi^{*}\right|+f(0)$ with $\xi^{*}=t_{0} \xi^{\prime}$. 
Then

$$
Q f\left(\xi^{*}\right)>P f\left(\xi^{*}\right) .
$$

Before proceeding to the proof, we make the following remarks.

Remarks 6.2. (a) By (6.1) the graph of $f$ and one of $C f$ intersect. This plays an important role in the proof of Theorem 6.1.

(b) Using Theorem 4.1, we can prove Theorem 6.1 only for $q$ near 2. But here we will conclude for every $q \in(1,2)$. This theorem implies $Q f_{p}>P f_{p}$ for every $p \in(1,2)$ where

$$
f_{p}(\xi)=\left\{\begin{array}{lll}
1+|\xi|^{p} & \text { if } & |\xi| \neq 0, \\
0 & \text { if } & |\xi|=0,
\end{array} \quad \xi \in \mathbb{R}^{2 \times 2} .\right.
$$

Note that in [9] Kohn and Strang proved that $P f_{2}=Q f_{2}$.

LeMmA 6.2. Let $N \geqq 1$ be an integer, $\Omega \subset \mathbb{R}^{N}$ a bounded open set, $\beta, \gamma>0$ and $r>1$. Let $\left(v_{n}\right)_{n} \subset L^{r}(\Omega)$ such that $\gamma \leqq\left\|v_{n}\right\|_{1},\left\|v_{n}\right\|_{r} \leqq \beta$ for all $n \in \mathbb{N}$. Then there exist $k, l>0$ such that $\left|\left\{x \in \Omega:\left|v_{n}(x)\right| \geqq k\right\}\right| \geqq$ l for every $n \in \mathbb{N}$.

Proof. The proof of Lemma 6.3 is elementary.

LEMMA 6.4. Let $f: \mathbb{R}^{2 \times 2} \mapsto \mathbb{R}$ a Borel measurable function, $a, b, c, \alpha>0, d \in \mathbb{R}$, $q \geqq 1$ and $\Omega \subset \mathbb{R}^{2}$ a bounded open set such that

$a|\xi|+f(0) \leqq f(\xi) \quad$ for every $\quad \xi \in \mathbb{R}^{2 \times 2} \quad$ with equality for $\quad \xi_{0}=\frac{\alpha}{\sqrt{2}}\left(\begin{array}{ll}1 & 0 \\ 0 & 1\end{array}\right) ;$

$$
b|\xi|^{q}+d \leqq f(\xi) \quad \text { for every } \quad \xi \in \mathbb{R}^{2 \times 2} .
$$

Then

$$
C f\left(t \xi_{0}\right)=a\left|t \xi_{0}\right|+f(0) \quad \text { for every } \quad t \in[0,1] .
$$

If for a fixed $\xi \in \mathbb{R}^{2 \times 2}$

$$
Q f(\xi)=a|\xi|+f(0)=\lim _{n \rightarrow \infty} \frac{1}{|\Omega|} \int_{\Omega} f\left(\xi+\nabla \phi_{n}\right),
$$

where $\left(\phi_{n}\right)_{n} \subset W_{0}^{1, \infty}(\Omega)^{2}$, then, up to a subsequence, the following hold:

$$
\begin{gathered}
\phi_{n} \rightarrow 0 W_{0}^{1, q}(\Omega)^{2}, \\
f\left(\xi+\nabla \phi_{n}\right)-a\left|\xi+\nabla \phi_{n}\right|-f(0) \rightarrow 0 L^{1}(\Omega), \\
\left|\xi+\nabla \phi_{n}\right|+|\xi|-\left|2 \xi+\nabla \phi_{n}\right| \rightarrow 0 L^{1}(\Omega) .
\end{gathered}
$$

But note that the following does not hold:

$$
\phi_{n} \rightarrow 0 W^{1,1}(\Omega) \text { if } f(\xi) \neq a|\xi|+f(0) \text { and } f \text { is continuous at } \xi .
$$

Proof. We first establish (6.7). We find using (6.5) that: $a|\xi|+f(0) \leqq C f(\xi)$ for every $\xi \in \mathbb{R}^{2 \times 2}$. Let $\xi \in \mathbb{R}^{2 \times 2}$ be such that $\xi=t \xi_{0}$ with $t \in[0,1]$. We have $C f(\xi) \leqq t f\left(\xi_{0}\right)+(1-t) f(0)=a|\xi|+f(0)$ and we obtain (6.7). 
We now prove (6.9), (6.10). Let $\xi \in \mathbb{R}^{2 \times 2}$ be fixed and $\left(\phi_{n}\right)_{n} \subset W_{0}^{1, \infty}(\Omega)^{2}$ such that $a|\xi|+f(0)=Q f(\xi)=\lim _{n \rightarrow \infty} \frac{1}{|\Omega|} \int_{\Omega} f\left(\xi+\nabla \phi_{n}\right)$. Using (6.6), we find that $\int_{\Omega}\left|\xi+\nabla \phi_{n}\right|^{q} \leqq \frac{1}{b}\left(\int_{\Omega}\left[f\left(\xi+\nabla \phi_{n}\right)-d\right]\right)$ and that $\left(\phi_{n}\right)_{n}$ is bounded in $W^{1, q}(\Omega)^{2}$. Up to a subsequence, we can suppose that

$$
\phi_{n} \underset{n \rightarrow \infty}{\stackrel{\text { weakly }}{\longrightarrow}} \phi W_{0}^{1, q}(\Omega)^{2} .
$$

This implies that

$$
\begin{aligned}
0 & \leqq \int_{\Omega}\left|f\left(\xi+\phi_{n}\right)-a\right| \xi+\nabla \phi_{n} \mid-f(0) \\
& =\int_{\Omega} f\left(\xi+\nabla \phi_{n}\right)-a\left|\xi+\nabla \phi_{n}\right|-f(0) \\
& \leqq \int_{\Omega} f\left(\xi+\nabla \phi_{n}\right)-a|\xi|-f(0) \rightarrow 0
\end{aligned}
$$

by (6.8). We therefore conclude that (6.10) is true. Using (6.8) we find $|\Omega|(a|\xi|+f(0))=\lim _{n \rightarrow \infty} \int_{\Omega} f\left(\xi+\nabla \phi_{n}\right)=\lim _{n \rightarrow \infty} \int_{\Omega}\left[a\left|\xi+\nabla \phi_{n}\right|+f(0)\right] \geqq \int_{\Omega}[a \mid \xi+$

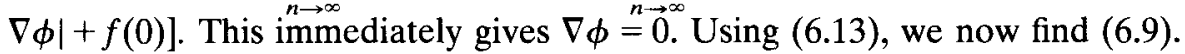

We now establish (6.11): up to a subsequence we can suppose that $\lim _{n \rightarrow \infty} \int_{\Omega}|2 \xi+\nabla \phi|$ exists. (6.9) implies that

$$
\int_{\Omega}|2 \xi| \leqq \lim _{n \rightarrow \infty} \int_{\Omega}|2 \xi+\nabla \phi| .
$$

Note that $f(\eta) \geqq a|\eta|+f(0)$ for every $\eta \in \mathbb{R}^{2 \times 2}$ and using (6.10) we obtain:

$$
\lim _{n \rightarrow \infty} \int_{\Omega}\left|\xi+\nabla \phi_{n}\right|=\int_{\Omega}|\xi|
$$

using (6.14) and (6.15) we find $0 \leqq \lim _{n \rightarrow \infty} \int_{\Omega}\left|\xi+\nabla \phi_{n}\right|+|\xi|-\left|2 \xi+\nabla \phi_{n}\right| \mid=\lim _{n \rightarrow \infty}$ $\int_{\Omega}\left|\xi+\nabla \phi_{n}\right|+|\xi|-\left|2 \xi+\nabla \phi_{n}\right| \leqq 0$. We therefore obtain (6.11).

We finally prove (6.12): assume that $f$ is continuous at $\xi$ and $f(\xi) \neq a|\xi|+f(0)$. Now we suppose that $\phi_{n} \rightarrow 0$ strongly in $W^{1,1}(\Omega)$ and show that this leads to a contradiction. Using a subsequence we can find an $x \in \Omega$ such that $\lim _{n \rightarrow \infty} \nabla \phi_{n}(x)=0$ and $\lim f\left(\xi+\nabla \phi_{n}(x)\right)-a\left|\xi+\nabla \phi_{n}(x)\right|-f(0)=0$. Thus $f(\xi)-a|\xi|-f(0)=0$, which $^{n}{ }_{\text {is }}^{\infty}$ a contradiction to our hypotheses. Thus, $\phi_{n} \rightarrow 0 W^{1,1}(\Omega)$ is false. This completes the proof of Lemma 6.3.

Proof of Theorem 6.1. The hypotheses on $f$ imply that $P f=C f$. To conclude, it suffices to show that $Q f\left(\xi^{*}\right)>C f\left(\xi^{*}\right)$. Recall that by (6.7) $C f\left(\xi^{*}\right)=a\left|\xi^{*}\right|+f(0)$. 
To obtain a contradiction, we suppose that $Q f\left(\xi^{*}\right)=a\left|\xi^{*}\right|+f(0)$. Assuming that

$$
u_{n}=2\left(\left|\partial_{2} \phi_{1}^{n}\right|+\left|\partial_{1} \phi_{2}^{n}\right|\right)+\left|\partial_{1} \phi_{1}^{n}-\partial_{2} \phi_{2}^{n}\right|, \quad \varepsilon \in(0, q-1) \quad \text { and } \quad v_{n}=u_{n}^{1+\varepsilon},
$$

we get $v_{n} \in L^{r}(\Omega)$, where $\partial_{1} \phi_{1}$ denotes $\partial \phi_{1} / \partial x_{1}$ and $r=q /(1+\varepsilon)>1$. Two cases may occur:

Case 1. $v_{n} \rightarrow 0 L^{1}(\Omega)$. It follows that $\Delta \phi_{n} \rightarrow 0 W^{-1,1 / \varepsilon}(\Omega)$, which implies that $\phi_{n} \rightarrow 0 W^{1,1+\varepsilon}(\Omega)$ and then $\phi_{n} \rightarrow 0 W^{1,1}(\Omega)$ (for more details see [13]). But by Lemma $6.4 \phi_{n} \rightarrow 0 W^{1,1}(\Omega)$ does not hold. We therefore have a contradiction.

Case 2. $v_{n} \rightarrow L^{1}(\Omega)$ does not hold. Using a subsequence, we then find that there exists a constant $\gamma>0$ such that, for every $n \in \mathbb{N}$, $\left\|v_{n}\right\|_{1} \geqq \gamma$. Since $\left(v_{n}\right)_{n}$ is bounded in $L^{r}(\Omega)$ and $r>1$, by Lemma 6.3, there exist two constants $\tilde{k}>0$ and $l>0$ such that $\left|\left\{x \in \Omega: v_{n}(x) \geqq \tilde{k}\right\}\right| \geqq l$ for all $n \in \mathbb{N}$. We immediately conclude that there exist $B>0, k>0$ such that $\mid\left\{x \in \Omega: u_{n}(x) \geqq k\right.$ and $\left.\left|\nabla \phi_{n}(x)\right|>B\right\} \mid \geqq l / 2$. We now write:

$$
\begin{gathered}
A_{n}=\left\{x \in \Omega: u_{n}(x) \geqq k,\left|\nabla \phi_{n}(x)\right| \leqq B\right\}, \\
K=\left\{\eta \in \mathbb{R}^{2 \times 2}:|\eta| \leqq B, 2\left(\left|\eta_{12}\right|+\left|\eta_{21}\right|\right)+\left|\eta_{11}-\eta_{22}\right| \geqq k\right\}, \\
F(\eta)=\frac{1}{2}|\xi+\eta|+\frac{1}{2}|\xi|-\frac{1}{2}|2 \xi+\eta| \eta \in \mathbb{R}^{2 \times 2} .
\end{gathered}
$$

$K$ is compact in $\mathbb{R}^{2 \times 2}, F$ is continuous in $\mathbb{R}^{2 \times 2}$ and we find $0<\beta=\min \{F(\eta): \eta \in K\}$ because $F(\eta) \leqq 0$ implies that $\eta=\left(\begin{array}{cc}\eta_{11} & 0 \\ 0 & \eta_{22}\end{array}\right) \notin K$. But $\int_{\Omega} F\left(\nabla \phi_{n}\right) \geqq \int_{A_{n}} F\left(\nabla \phi_{n}\right) \geqq \beta\left|A_{n}\right| \geqq \frac{l \beta}{2}$. Furthermore, using (6.11) in Lemma 6.4, we obtain $0=\lim _{n \rightarrow \infty} \int_{\Omega} F\left(\nabla \phi_{n}\right)$, a contradiction. We therefore deduce that $v_{n} \rightarrow 0$ $L^{1}(\Omega)$. Since the two cases do not apply, we conclude that

which is equivalent to

$$
Q f\left(\xi^{*}\right) \neq C f\left(\xi^{*}\right)
$$

$$
Q f\left(\xi^{*}\right)>P f\left(\xi^{*}\right)
$$

This finishes the proof of Theorem 6.1.

Corollary 6.5. Let $p \in(1,2), t \in(0, \alpha), \alpha=[1 /(p-1)]^{1 / p}, \xi_{t}=\frac{t}{\sqrt{2}}\left(\begin{array}{ll}1 & 0 \\ 0 & 1\end{array}\right)$ and

Then

$$
f_{p}(\xi)=\left\{\begin{array}{lll}
1+|\xi|^{p} & \text { if } & |\xi| \neq 0, \\
0 & \text { if } & |\xi|=0,
\end{array} \quad \xi \in \mathbb{R}^{2 \times 2}\right.
$$

$$
P f_{p}\left(\xi_{t}\right)<Q f_{p}\left(\xi_{t}\right)
$$

Proof. It is easy to see that

$$
C f_{p}(\xi)=\left\{\begin{array}{lll}
1+|\xi|^{p} & \text { if } & |\xi| \geqq \alpha, \\
a|\xi| & \text { if } & |\xi|<\alpha,
\end{array}\right.
$$

where $a=p^{1 / p} p^{1 / p^{\prime}}$ and $p^{\prime}=p /(p-1)$. Additionally the following relations hold:

(a) $R f_{p}(\xi)=Q f_{p}(\xi)=P f_{p}(\xi)=C f_{p}(\xi)=f(\xi)$ for every $|\xi| \geqq \alpha$; 
(b) $a|\xi| \leqq f(\xi)$ with equality if and only if $|\xi|=0$ or $\alpha$;

(c) $0<\left|\xi_{t}\right|=t<\alpha$.

These are the hypotheses of Theorem 6.1. Thus, Corollary 6.5 is proved.

Remark 6.6. To construct a function $f: \mathbb{R}^{2 \times 2} \mapsto \mathbb{R}$ satisfying the hypotheses of Theorem 6.1, it suffices to construct a continuous function $g:[0, \infty[\rightarrow \mathbb{R}$ such that:

$$
g(0)=0, \quad g(x)=1+x^{p} \quad \text { if } \quad x \geqq \alpha \quad a x<g(x)<1+x^{p} \quad \text { if } \quad x \in(0, \alpha),
$$

where

$$
1<p<2, \quad a=p^{1 / p} p^{\prime 1 / p^{\prime}}, \quad p^{\prime}=\frac{p}{p-1}, \quad \text { and } \quad \alpha=\left[\frac{1}{p-1}\right]^{1 / p} .
$$

Assuming that $f(\xi)=g(|\xi|)$, we find that

$$
Q f\left(\xi_{t}\right)>P f\left(\xi_{t}\right)
$$

for every $\xi_{t}=\frac{t}{\sqrt{2}}\left(\begin{array}{ll}1 & 0 \\ 0 & 1\end{array}\right), t \in(0, \alpha)$.

\section{Acknowledgments}

I would like to thank L. Boccardo, G. Buttazo and B. Dacorogna for discussion and encouragement, and G. Manogg for criticism of the manuscript.

\section{References}

1 J. J. Alibert and B. Dacorogna. An example of a quasiconvex function that is not polyconvex in two dimension. Arch. Rational Mech. Anal. 117 (1992) 155-166.

2 J. M. Ball. Convexity conditions and existence theorems in nonlinear elasticity. Arch. Rational Mech. Anal. 64 (1977), 337-403.

3 H. Brezis. Analyse Fonctionnelle (Paris: Masson, 1983).

4 B. Dacorogna. Direct Methods in the Calculus of Variations (Berlin: 1989).

5 I. Ekeland. Non convex minimization problem. Bull. Amer. Math. Soc. 1 (3) (1979) 443-474.

6 F. Gehring. The $L^{p}$ integrability of the partial derivatives of quasiconformal mapping. Acta Math. 130 (1973), 265-277.

7 M. Giaquinta and G. Modica. Regularity results for some classes of higher order non linear elliptic systems. J. Reine Angew. Math. 311/312 (1979), 145-169.

8 R. V. Kohn. The relaxation of a double-well energy (to appear).

9 R. V. Kohn and G. Strang. Optimal design and relaxation of variational problems I, II and III. Comm. Pure Appl. Math. 39 (1986), 113-137; 139-182; 353-377.

10 P. Marcellini and C. Sbordone. On the existence of minima of multiple integrals. J. Math. Pures Appl. 62 (1983), 1-9.

11 C. B. Morrey. Quasiconvexity and semicontinuity of multiple integrales. Pacific J. Math. 2 (1952), 25-53.

12 C. B. Morrey. Multiple Integrals in the Calculus of Variations (Berlin: Springer, 1966).

13 C. G. Simader. On Dirichlet's Boundary Value Problem, Lecture Notes in Math. 268 (Berlin: Springer, 1972).

14 V. Sverak. Quasiconvex functions with subquadratic growth (to appear).

(Issued 11 August 1993) 